\title{
超分子有机框架对喜树碱类开环羧酸盐的负载及其内酯化动力学
}

\author{
间萌彭文昶王辉张丹维黎占亭* \\ (复旦大学化学系 上海 200438)
}

\begin{abstract}
摘要 喜树碱在弱碱性生理介质中主要以无生物活性的开环羧酸盐形式存在, 肿瘤微环境具有弱酸性, 而超分子有机 框架可以富集和输送负离子药物至肿瘤微环境和肿瘤细胞内. 为评估通过超分子有机框架富集和输送喜树碱类开环羧 酸盐到肿瘤微环境及肿瘤细胞内, 利用肿瘤弱酸性微环境驱动其内酯化, 从而发展羧酸盐为前药的可能性, 利用透析 实验研究了两个超分子有机框架对喜树碱, SN-38 和 10-差基喜树碱开环羧酸盐负离子的吸收和保留效应, 利用吸收光 谱研究了三个羧酸盐在弱酸性生理盐水介质中内醌化形成活性喜树碱分子的动力学. 结果表明超分子有机框架对三个 羧酸盐均具有显著的保留效应, 在 $\mathrm{pH}$ 为 6.5 的弱酸性介质中, 羧酸盐转化为相应内酯的(表观)半衰期分别为 120,22 和 $31 \mathrm{~h}$, 远短于临床应用的喜树碱类药物伊立替康和拓扑替康重复用药间隔时间 $(14$ 和 $21 \mathrm{~d})$, 为后续研究提供了依据. 关键词 喜树碱; SN-38; 10-羟基喜树碱; 开环羧酸盐; 超分子有机框架; 负载; 内酯化动力学
\end{abstract}

\section{Supramolecular Organic Framework Loading for Camptothecin Open-Ring Carboxylates and Their Lactonization Kinetics}

\author{
Yan, Meng \\ Peng, Wenchang \\ Wang, Hui \\ Zhang, Danwei \\ (Department of Chemistry, Fudan University, Shanghai 200438)
}

Li, Zhanting*

\begin{abstract}
Camptothecin derivatives exist mainly as inactive open ring carboxylates in physiological media. Tumor microenvironment is generally weakly acidic, whereas supramolecular organic frameworks can adsorb and deliver anionic antitumor drugs into tumor cells. In principle, supramolecular organic frameworks (SOFs) can adsorb and deliver camptothecin open ring carboxylates to tumor microenvironment and the delivered carboxylates can undergo lactonization to afford active molecules due to the weak acid character of the tumor microenvironment, which may lead to efficient utilization of the carboxylates as predrugs. To explore this potential, dialysis experiments for the open ring carboxylates of camptothecin, SN-38 and 10-hydroxycamptothecin in the presence of two SOFs were conducted, which revealed important adsorption and retaining capacity of the SOFs for the carboxylates. Absorption spectroscopy was also utilized to evaluate the lactonization kinetics of the three carboxylates in weakly acidic saline solution. The result reveals that at $\mathrm{pH}=6.5$, the half lives of the conversion are 120,22 and $31 \mathrm{~h}$, respectively, which are considerably shorter than the time gap for repeated administrations of clinically used irinotican (14 d) or topotecan ( $21 \mathrm{~d})$, which provides the experimental basis for further study.

Keywords camptothecin; SN-38; 10-hydroxycamptothecin; open ring carboxylate; supramolecular organic frameworks; loading; lactonization kinetics
\end{abstract}

喜树碱类有机分子具有显著的抗肿瘤作用 ${ }^{[1]}$, 但喜 树碱 (camptothecin, CPT) 及活性更高的修饰物，如 SN-38 (7-ethyl-10-hydroxycamptothecin)和 10-羟基喜树 碱(10-hydroxycamptothecin, 10-HCPT)等，水溶性都很 低 ${ }^{[2]}$, 并具有不同的副作用 ${ }^{[3]}$, 骨架中六元环内酯在生 理环境中 $(\mathrm{pH}=7.4)$ 也都易开环, 主要以相应的无活性 的羧酸盐形式存在 ${ }^{[4]}$. 因此, 尽管很多此类骨架衍生物
得到长期研究, 但仍难以获批进入临床. 目前只有拓扑 替康(Topotecan)作为 CPT 半合成衍生物在临床上用于 治疗卵巢癌等癌症, 伊立替康(Irinotecan)作为活性分子 SN-38 的前药在临床上用于治疗结肠癌等. 两个药物在 体内都主要开环为无活性的羧酸盐, 生物利用度很低 ${ }^{[5]}$. CPT 本身天然资源丰富, 修饰合成研究深入, 获得的活 性分子也数量众多 ${ }^{[6]}$. 进一步发展有效途径、克服此类

\footnotetext{
* Corresponding author. E-mail: ztli@fudan.edu.cn

Received March 29, 2019; revised April 19, 2019; published online April 26, 2019

Project supported by the National Natural Science Foundation of China (Nos. 21432004, 21529201, 21890732).

国家自然科学基金(Nos. 21432004, 21529201, 21890732)资助项目.
} 
分子的低水溶性、提高生物利用度或通过降低用量降低 副作用等都有助于进一步拓展 CPT 类分子的临床应用. 目前国内外研究的一个重点是, 发展脂质体和各种纳米 载体输送活性分子或以偶联前药方式输送 ${ }^{[7]}$, 一些载体 输送型前药如 NK012 等进入了临床前研究 ${ }^{[8]}$, 只有伊立 替康脂质体获批进入临床使用 ${ }^{[9]}$. 由于在正常生理介质 $(\mathrm{pH} \approx 7.4)$ 中, $\mathrm{CPT}$ 类分子主要以内酯开环羧酸盐形式存 在, 研究发现有效利用这些无生物活性的羧酸盐的新方 法将具有潜在的应用价值.

我们研究组最近利用自组装策略, 通过多臂正离子 四面体单体和葫芦䐂 $[8]$ 的高强度结合 ${ }^{[10]}$, 构建了水溶 性的三维空穴超分子有机框架(Supramolecular organic frameworks, SOFs $)^{[11,12]}$. 作为一类由葫芦脲包结构筑的 具有正离子型聚电解质特征的特殊有序超分子聚合 物 ${ }^{[13]}, \mathrm{SOF}$ 可以通过静电作用吸收负离子药物培美曲塞 等, 并具有纳米粒效应, 可以实现对耐药性乳腺肿瘤细 胞的药物输送及细胞生长抑制 ${ }^{[14]}$. 无抗肿瘤活性的 CPT 类羧酸盐负离子理论上可以被 SOF 吸收和输送 ${ }^{[13 \sim 15]}$. 肿瘤微环境具有弱酸性 ${ }^{[16]}$, 大量研究已经确立, 在酸性 介质中, $\mathrm{CPT}$ 类羧酸盐的 $\mathrm{CO}_{2} \mathrm{H}$ 与 17- $\mathrm{OH}$ 缩合形成环内 酯 ${ }^{[17]}$, 转化为活性分子本身. 这一转化为利用 SOF 开展 $\mathrm{CPT}$ 类羧酸盐输送和肿瘤细胞内活性分子结构恢复及 肿瘤治疗提供了可能. 为探索这一可能性, 需要系统研 究 SOF 对这些羧酸盐的吸收和保留作用及这些羧酸盐 在不同弱酸性介质中成环形成环内酯的速率. 基于上述 考虑, 我们利用透析方法系统地研究了两个 SOF 载体 对三个喜树碱羧酸盐 CPT-c, SN-38-c 和 10-HCPT-c 的吸 收保留效应, 利用吸收光谱研究了三个羧酸盐在不同弱 酸性介质中成环转化为活性分子 $\mathrm{CPT}, \mathrm{SN}-38$ 和 10-HCPT 的动力学 (Eq. 1), 为进一步开展后续输送研究 提供了实验依据.

\section{1 结果与讨论}

化合物 CPT-c 为商品试剂, SN-38-c 和 10-HCPT-c 分别由 SN-38 和 10-HCPT 水解制备. CPT, SN-38 和 10-HCPT 不溶于水, 而 CPT-c, SN-38-c 和 10-HCPT-c 具 有水溶性, 其中 CPT-c 为单价羧酸盐, SN-38-c 和 10-HCPT-c 为二价(羧酸和酚)钠盐. 我们之前研究表明, 三维 SOF 作为正离子型自组装孔结构, 其正离子吡啶 盐与配对负离子 $\mathrm{Cl}^{-}$或 $\mathrm{Br}^{-}$形成软酸-硬碱离子对, 在水 中与有机负离子钠盐或钾盐(硬酸-软碱离子对)发生离 子交换, 吸收有机负离子软碱到其内部, 产生富集效 应 $^{[12 \mathrm{a}, 14,15]}$. 为此, 我们选择由外侧并入 4 -苯基吡啶(4phenylpyridine, pp)和 1,4-二吡啶苯 [1,4-bis(pyridin-4yl)benzene, bpb]片段的四面体单体 T-pp 和 T-bpb 与 CB

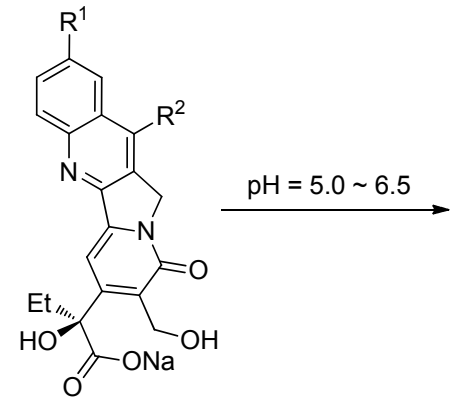

CPT-C: $\mathrm{R}^{1}=\mathrm{R}^{2}=\mathrm{H}$

SN-38-C: $R^{1}=\mathrm{ONa}, \mathrm{R}^{2}=\mathrm{Et}$

10-HCPT-C: $\mathrm{R}^{1}=\mathrm{ONa}, \mathrm{R}^{2}=\mathrm{H}$

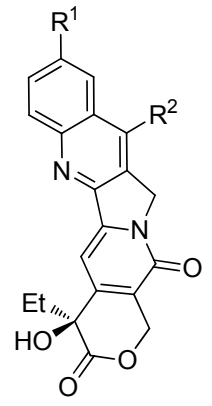

CPT

$\mathrm{SN}-38$

10-HCPT
[8]结合构筑的两个超分子有机框架 SOF-pp 和 SOF$\mathrm{bpb}\left(\right.$ 图 1) ${ }^{[12 a, 18]}$ 作为吸收 CPT 开环羧酸盐的载体. SOF-pp 和 SOF-bpb 分别形成内径为 2.1 和 $3.6 \mathrm{~nm}$ 的类似环已烷 椅式构象的六边形孔径，已被揭示可以吸收富集负离子 药物和过渡金属络合物等 ${ }^{[12 a, 18]}$.

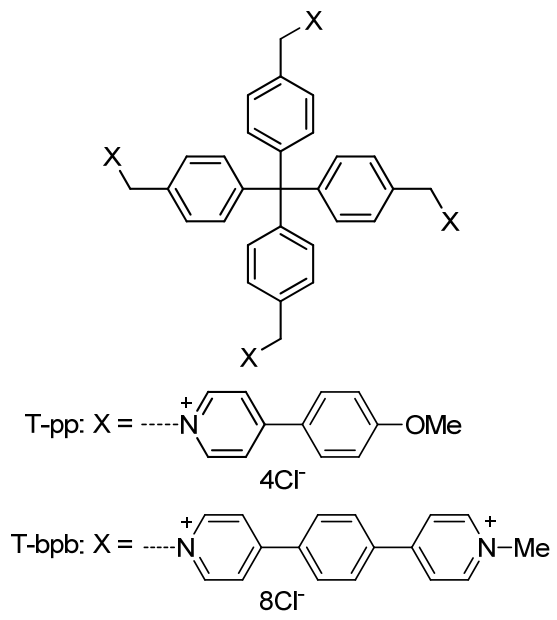

图 1 构筑 SOF-pp 和 SOF-bpb 的四面体单体 T-pp 和 T-bpb Figure 1 Structures of building blocks T-pp and T-bpb for SOF-pp and SOF-bpb

透析实验用于揭示 SOF-pp 和 SOF-bpb 对三个羧酸 盐的富集和保留效应. 实验在生理盐水中和人体正常温 度 $37{ }^{\circ} \mathrm{C}$ 下进行, 通过摇床固定震荡速率保证透析条件 一致. 从透析袋中透析到外面生理盐水中的样品, 通过 吸收光谱测定样品特征吸收带吸光度, 与标准样品比对 计算获得透析出的样品的相对比例. 吸收光谱稀释实验 表明, 在所研究的浓度范围内, 三个羧酸盐的吸收光谱 的吸光度都与浓度呈线性关系, 表明其以单分子状态存 在，没有发生簇集. 使用截留分子量为 $1000 \mathrm{D}$ 的透析袋 保证羧酸盐透过，而吸收光谱显示 SOF 单体 T-pp 和 T-bpb 及 $\mathrm{CB}[8]$ 不能有效通过. 在测试时间范围内, 透析 到外侧溶液的这些样品的吸光度低于 0.005 , 可以忽略 不计. 为便于比较, 所有实验的 SOF 与羧酸盐的质量比 均为 $10: 1$, 整个透析体系溶剂体积均为 $30 \mathrm{~mL}$ (透析袋 
内外溶液总体积). 对于透析实验(包括控制实验), 透析 袋内溶液体积为 $4 \mathrm{~mL}$, 其中 CPT-c, SN-38-c 和 $10-\mathrm{HCPT}-\mathrm{c}$ 都为 $1 \mathrm{mg}$, SOF 载体为 $10 \mathrm{mg}$.

图 2 为两个 SOF 存在下不同时间内透析到外侧溶 液中的三个羧酸盐的吸收光谱, 图 3 为包括不加 SOF 的 相应的透析率(透析样品与总样品的比值)随时间的变化 趋势. 不加 SOF 载体, CPT-c 在大约 $16 \mathrm{~h}$ 后透析达到平 衡(透析袋内外浓度相同), 即透析袋内外 CPT-c 浓度相 同, 透出约 $88 \%$, 而在约 $1.5 \mathrm{~h}$ 透出即达到 $50 \%$ (图 3a). 在 SOF-pp 和 SOF-bpb 存在下, CPT-c 透出速率明显下降, 两个载体具有明显的保留效应. 在 SOF-pp 存在下, 8 和 $16 \mathrm{~h}$ 后分别透出 $46 \%$ 和 54\%的 CPT-c, 即透析袋内分别 保留 $54 \%$ 和 $46 \%$. 而在 SOF-bpb 存在下, 8 和 $16 \mathrm{~h}$ 后, CPT-c 透出分别约 33\% 和 46\%, 即有 $67 \%$ 和 54\%的保留.
在所有时间段, SOF-bpb 的保留效果都更显著.

在不加 SOF 的情况下, SN-38-c 在约 $4 \mathrm{~h}$ 即透出一 半, 在约 $13 \mathrm{~h}$ 达到透析平衡(图 3b). 在 SOF-pp 存在下, 其在 $5 \mathrm{~h}$ 后大约透出 $25 \%$, 而透出 $50 \%$ 需要 $22 \mathrm{~h}$, 总体 展示出较强的保留效应. SOF-bpb 展示出更高的保留效 果. 在 $5 \mathrm{~h}$ 后, 透出率仅为 $4.8 \%$, 而在最长的测定时间 $36 \mathrm{~h}$, 也仅有 $20 \%$ 的样品透出. 而不加 SOF 的 10-HCPT-c 样品, $5 \mathrm{~h}$ 透出约 $50 \%$, 在 $24 \mathrm{~h}$ 透析达到平衡. 在 SOF-pp 存在下, $5 \mathrm{~h}$ 大约有 $25 \%$ 样品透出, $24 \mathrm{~h}$ 后, 透 出约 $49 \%$. 同样条件下, 在 SOF-bpb 存在下, $5 \mathrm{~h}$ 后其透 出率降低至约 $9 \%$, 而在 $24 \mathrm{~h}$ 后透出率也降低, 约为 $33 \%$. 上述结果显示, 两个 SOF 结构都能保留三个羧酸 盐, 其中 SOF-bpb 保留效果更加显著.
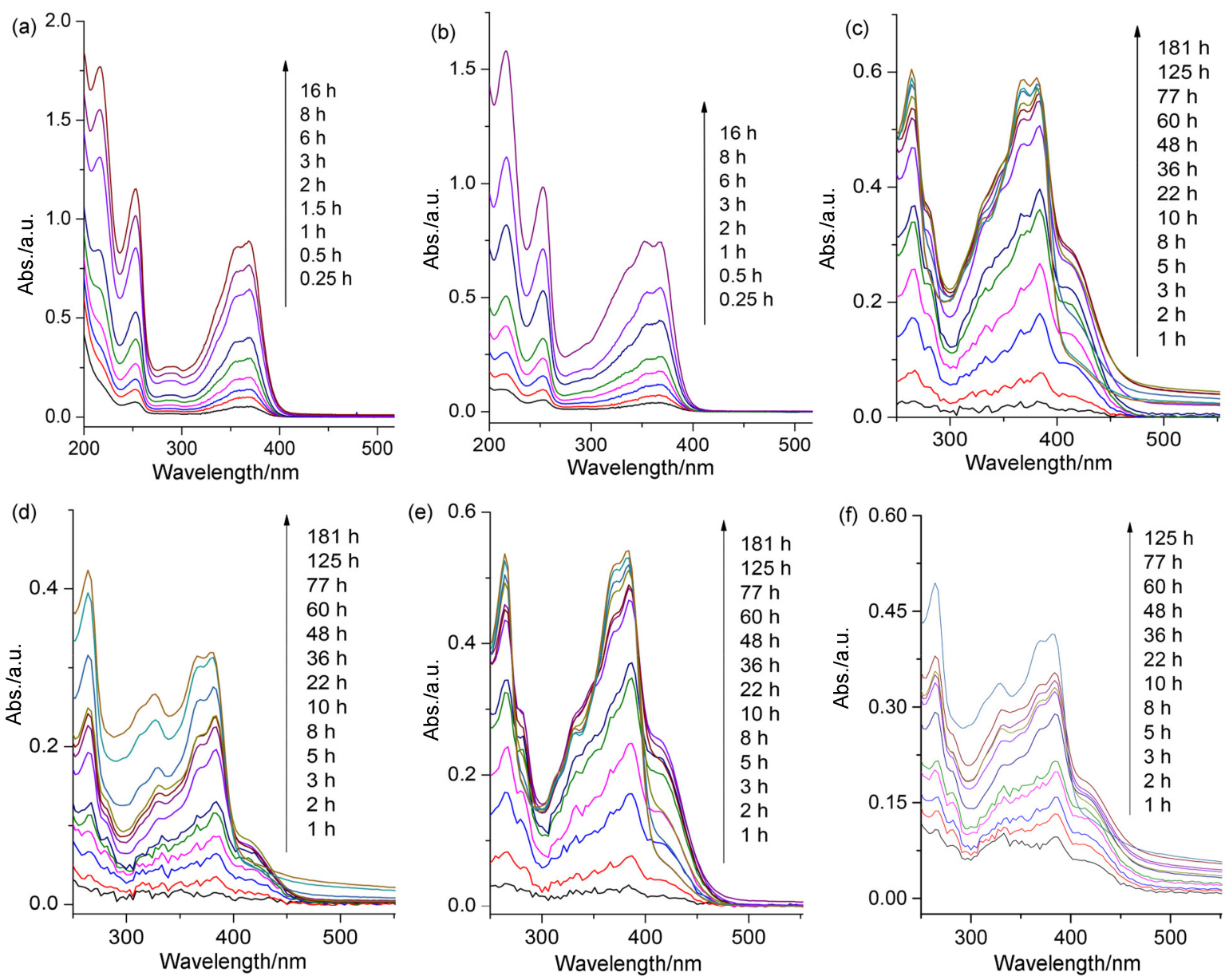

图 2 时间依赖的透析液吸收光谱 $(0.9 \%$ sodium chloride solution in water)

Figure 2 Time-dependent absorption spectra of the outside solution of the dialysis experiments ( $0.9 \%$ sodium chloride solution in water)

Samples in dialysis bags: (a) CPT-c (1 mg) + SOF-pp (10 mg), (b) CPT-c (1 mg) + SOF-bpb (10 mg), (c) SN-38-c (1 mg) + SOF-pp (10 mg), (d) SN-38-c $(1 \mathrm{mg})+$ SOF-bpb (10 mg), (e) 10-HCPT-c (1 mg) +SOF-pp (10 mg), and (f) 10-HCPT-c (1 mg) +SOF-bpb (5 mg). For all samples: solution volume in dialysis bag: $4 \mathrm{~mL}$, outside solution: $26 \mathrm{~mL}$, dialysis temperature: $37{ }^{\circ} \mathrm{C}$, spectra recorded at $25^{\circ} \mathrm{C}$. 

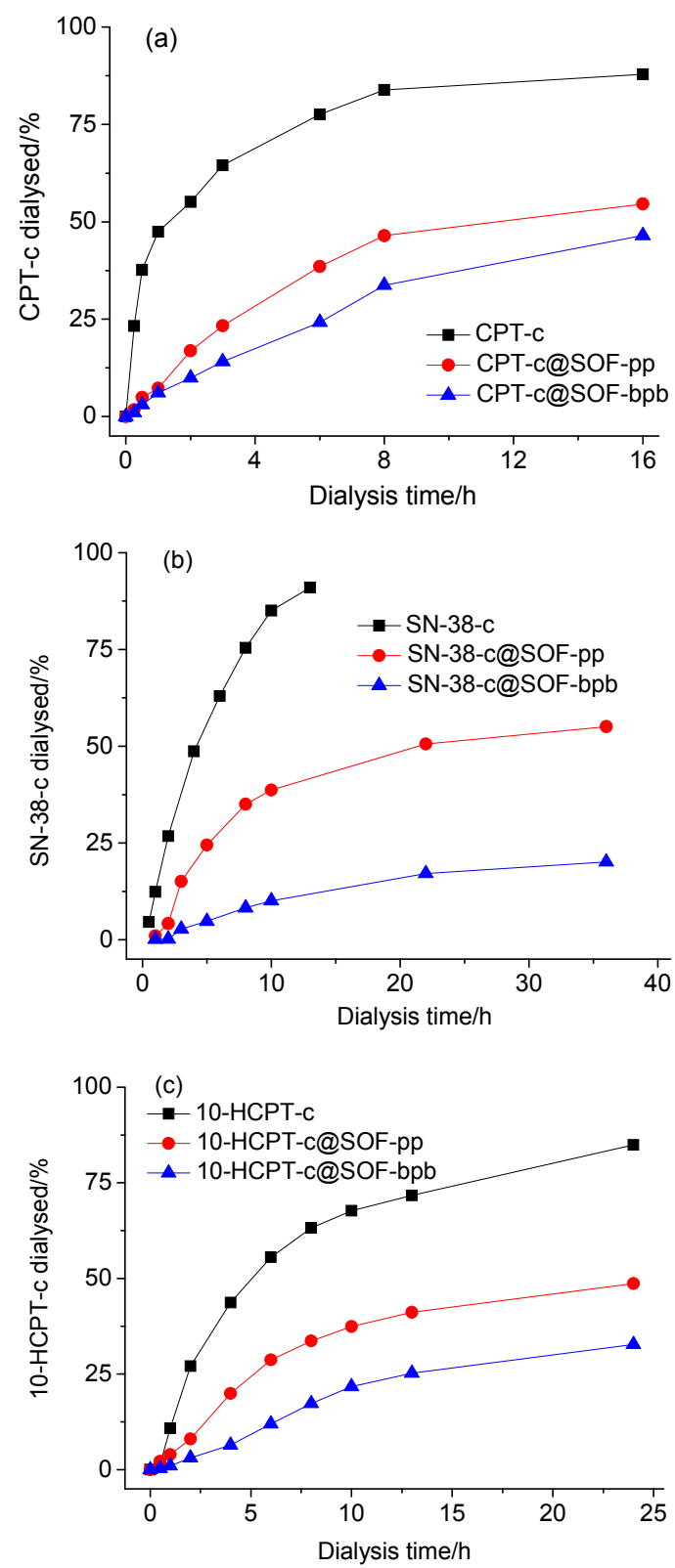

图 3 喜树碱开环羧酸盐在没有和 SOF-pp 或 SOF-bpb 存在下 的时间依赖的透析率

Figure 3 Time-dependent dialysis percentage of camptothecin open ring carboxylates in the absence and presence of SOF-pp or SOF-bpb

(a) CPT-c, (b) SN-38-c and (c) 10-HCPT-c. For all samples: carboxylate $(1 \mathrm{mg})$ with or without SOF $(10 \mathrm{mg})$; solution volume in dialysis bag: 4 $\mathrm{mL}$, outside solution: $26 \mathrm{~mL}$, dialysis temperature: $37^{\circ} \mathrm{C}$, spectra recorded at $25{ }^{\circ} \mathrm{C}$.

上述透析实验表明, SOF 对三个羧酸盐都具有明显 的保留效应. 由于 SOF 作为载体的输送效应已经比较 明确 ${ }^{[14]}$, 进一步地系统研究不同弱酸性介质中羧酸盐 转化为活性内酯分子的动力学非常重要. 这方面的结果 将可以帮助评估基于 SOF 输送的非活性羧酸盐的应用 潜力. 为此进一步研究了三个喜树碱开环羧酸盐在弱酸 性生理盐水 $(0.9 \%$ 氯化钠十盐酸)中内酯化形成活性分子
的动力学. 由于羧酸盐具有水溶性，而活性分子本身水 溶性极低, 可以通过记录羒酸盐的吸收光谱, 研究这一 一级动力学反应. 对于记录的所有光谱, 假设内酯分子 已达到饱和, 进一步产生的产物析出. 通过减去内酯在 饱和溶液中的吸收光谱, 确定未转化羧酸盐的浓度. 为 揭示不同弱酸性介质下的转化差异, 反应分别在 $\mathrm{pH}=$ $5.0,5.5,6.0$ 和 6.5 介质中进行. 事实上, 三个内酯分子 在生理盐水介质中的饱和溶液的吸收光谱都非常弱, 在 选择的读取羧酸盐吸光度的波长其吸光度都小于 0.005 , 因此可以做忽略不计处理. 所有样品都配成起始浓度为 $50 \mu \mathrm{g} / \mathrm{mL}$ 的具有上述四个 $\mathrm{pH}$ 值的生理盐水溶液, 在 $37{ }^{\circ} \mathrm{C}$ 放置, 在一定时间后每次取 $2.5 \mathrm{~mL}$ 在 $25{ }^{\circ} \mathrm{C}$ 记录 吸收光谱. 所有样品在最初阶段都保持透明, 表明相应 的羧酸具有足够的溶解性.

在所有四个不同 $\mathrm{pH}$ 值的弱酸性介质中, CPT-c 的溶 液的吸收光谱都随时间逐渐变弱. 把 $364 \mathrm{~nm}$ 处吸光度 随时间变化的数据用一级反应动力学处理, 可以得到相 应酯化反应的半衰期 $\left(t_{1 / 2}\right.$, 表 1$)$. 可以看出, 随着 $\mathrm{pH}$ 从 6.5 减低到 5.0, 即酸性逐渐增强, 其内酯化半衰期从 $120 \mathrm{~h}$ 缩短到 $72 \mathrm{~h}$. SN-38-c 的溶液的吸收光谱在最初的 $10 \mathrm{~h}$ 表现出复杂的变化. 其最初形成的羧酸(包括吡啶 N 质子化形式)吸收峰逐渐降低(特征吸收峰: 281,341 和 $413 \mathrm{~nm}$, 图 4a), 而另一套吸收峰逐渐增强 $(265,370$ 和 $383 \mathrm{~nm}$ ). 随着时间进一步延长, 这一套特征吸收峰又 逐渐变弱(图 4b), 但峰型不变, 表明其由单一物种产生, 逐渐转化为不溶性内酯产物 SN-38. 在第一时间段逐渐 增强的吸收峰表明, 在形成最终的不溶性内酯之前, 羒 酸盐质子化后首先形成了一个新的中间体，但目前尚不 能确定其结构. 因为本项研究使用羧酸盐纯品, 吡啶 $\mathrm{N}$ 和羧酸盐质子化都是一个快速步骤，而在第一阶段又没 有沉淀产生，排除了活性内酯分子的形成，一种可能是 吡啶 $\mathrm{N}$ 质子化诱导多环骨架异构化, 形成共平面的喹啉 并吲哚嗪异构体. 10-HCPT-c 也表现出类似现象(图 4c), 但第一阶段光谱吸收变化较小, 并在约 $8 \mathrm{~h}$ 达到拐点, 之后光谱形状不再发生变化, 并逐渐减弱(图 4d), 表明 相应中间体进一步形成了不溶性的内酯分子 10-HCPT. 尽管 CPT-c 没有出现上述吸光度增加的现象, 但在约 $12 \sim 15 \mathrm{~h}$ 后也出现一个可观察到的拐点, 表明也可能形 成了类似的新的中间体, 但吸收光谱变化幅度较小.

由于 SN-38-c 和 10-HCPT-c 在第一阶段形成了新的 中间体产物，这一阶段预期又同时形成微量的内酯化产 物 SN-38 和 10-HCPT, 我们只对在第二阶段转化为 SN-38 和 10-HCPT 的过程作一级动力学分析, 利用不同 时间的吸光度求得这一转化过程的半衰期, 结果也见表 1. 从表中可以看出, 转化为 $\mathrm{SN}-38$ 的半衰期在不同 $\mathrm{pH}$ 

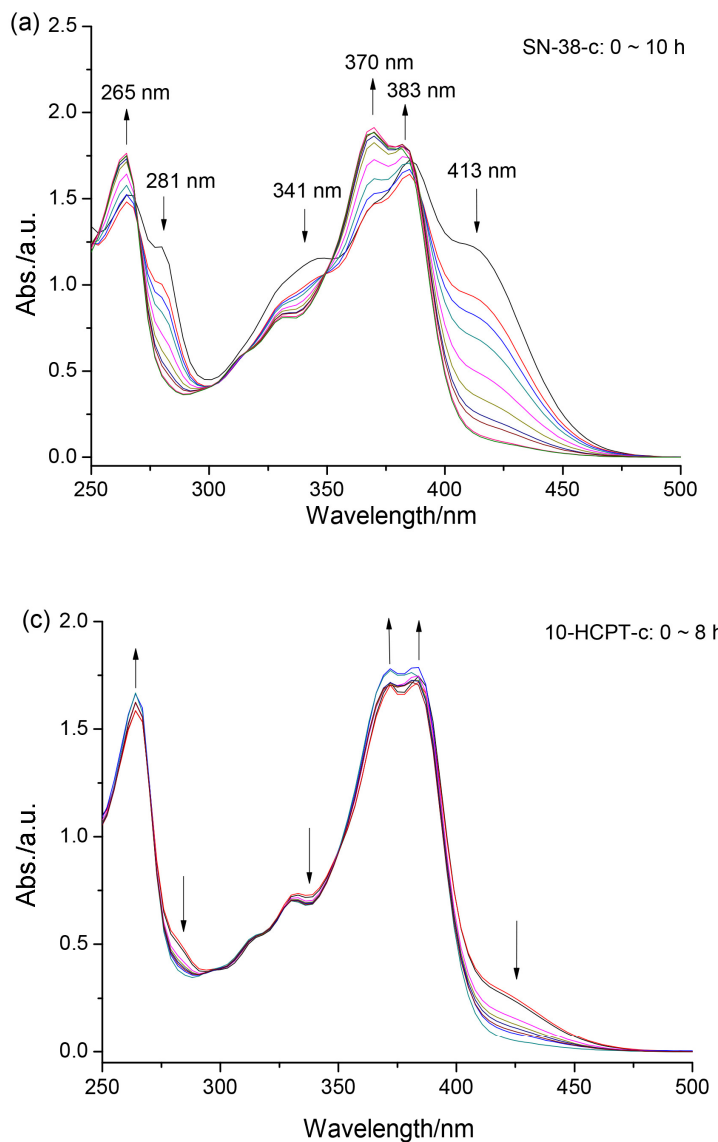
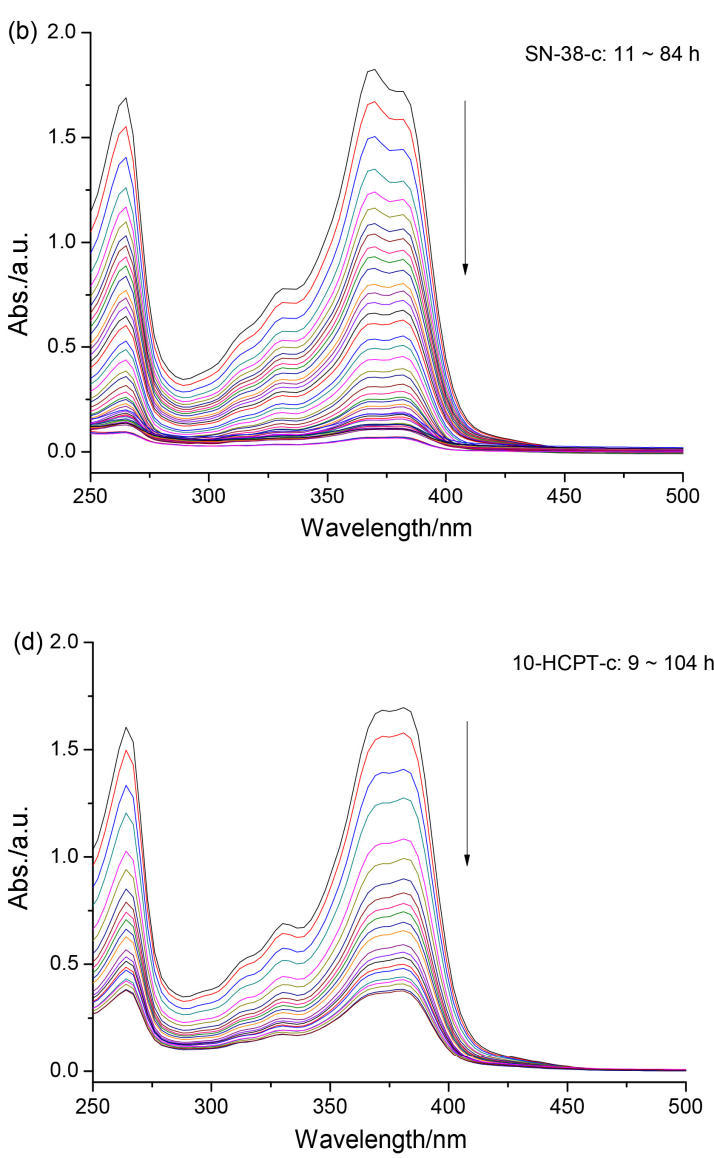

图 4 SN-38-c ( $\mathrm{a}$ 和 $\mathrm{b})$ 和 10-HCPT-c (c 和 $\mathrm{d})$ 在生理盐水 $(\mathrm{pH}=5.0, \mathrm{HCl})$ 中的吸收光谱

Figure 4 Absorbance spectra of SN-38-c (a and b) and 10-HCPT-c (c and d) in saline solution $(\mathrm{pH}=5.0, \mathrm{HCl})$ Concentration of both samples was $50 \mu \mathrm{g} / \mathrm{mL}$, the samples were placed at $37{ }^{\circ} \mathrm{C}$ and the spectra were recorded at $25{ }^{\circ} \mathrm{C}$

表 1 喜树碱开环羧酸盐在不同弱酸性生理盐水介质中内酯 化反应的半衰期 $\left(t_{1 / 2} / \mathrm{h}\right)^{a}$

Table 1 Half life $\left(t_{1 / 2} / \mathrm{h}\right)$ of the lactonization of the camptothecin open ring carboxylates in weakly acidic physiological saline solution

\begin{tabular}{cccc}
\hline $\mathrm{pH}^{b}$ & CPT-c & SN-38-c & 10-HCPT-c \\
\hline 5.0 & 72 & 10.9 & 18.5 \\
5.5 & 83 & 10.7 & 19.2 \\
6.0 & 104 & 10.9 & 22.8 \\
6.5 & 120 & 11.7 & 23.1 \\
\hline
\end{tabular}

${ }^{a}$ In $0.9 \%$ saline solution tuned by hydrochloric acid. ${ }^{b}$ At $37{ }^{\circ} \mathrm{C}$.

下都比 10-HCPT 要短, 两者的数据有都明显短于 CPT-c 形成 CPT 的内酯化反应. 降低 $\mathrm{pH}$ 值增加酸性, 所有三 个样品转化为内酯活性分子的速率有所增加, 尽管 CPT-c 增加幅度最大, 但三个样品半衰期缩短都在一倍 以内. 如果考虑到第一阶段形成中间体产物的时间, 两 个羧酸盐 $50 \%$ 转化为相应的内酯产物最多需要 22 和 31 h. 而伊立替康用药间隔为 $14 \mathrm{~d}$, 拓扑替康连续 $5 \mathrm{~d}$ 用药 后再次用药间隔为 $21 \mathrm{~d}$. 即使 CPT-c 内酯化半衰期最长 为 $5 \mathrm{~d}$, 这些定量评估揭示, 所有三个羧酸盐的内酯化 半衰期也都明显短于伊立替康和拓扑替康间隔重复用
药时间, 值得进一步研究 SOF 对其输送及评估这些羧 酸盐可能的前药功能.

\section{2 结论}

通过透析实验揭示, 超分子有机框架对喜树碱开环 羧酸盐具有显著的吸收富集和保留作用, 通过吸收光谱 实验揭示了开环䊣酸盐在弱酸性介质中较快转化为喜 树碱类活性分子, (表观)半衰期远短于临床应用的伊立 替康和拓扑替康重复用药周期. 尽管 SN-38-c 和 10-HCPT-c 内酯化过程中形成了具有一定稳定性的中间 体化合物, 研究结果表明, 如果能发展有效途径, 实现 对开环羧酸盐的肿瘤微环境和细胞内输送, 有可能利用 肿瘤微环境的弱酸性特征实现非活性的羧酸盐作为前 药的有效利用.

\section{3 实验部分}

\section{1 仪器与试剂}

溶剂和试剂使用前没有进一步纯化. CPT, SN-38, HO-HCPT 和 CPT-c 分别购自 Tokyo Chemical Industry 
Co., Bide Pharmatech (Shanghai), Sangon Biotech (shanghai)和 Aldrich-Sigma 公司. 核磁在 400 兆 Bruker Avance III HD 型核磁共振仪记录, 质谱在 Bruker McriOTOF 11 型质谱仪测试, 吸收光谱在 Perkin Almer 750s 型分光光 度计记录。

\section{2 化合物合成}

氮气保护下, 把化合物 SN-38 (0.39 g, $1.0 \mathrm{mmol})$ 加 入到 $2 \mathrm{~mL}$ 氢氧化钠 $(80 \mathrm{mg}, 2.0 \mathrm{mmol})$ 水溶液中. 室温摚 拌 $2 \mathrm{~h}$ 后用二氯甲烷萃取 $(2 \mathrm{~mL} \times 3)$. 水溶液用旋转蒸发 仪浓缩干燥后得黄色固体 SN-38-c $0.45 \mathrm{~g}$, 产率 $99 \%$. m.p. $>300{ }^{\circ} \mathrm{C}$ (dec.). ${ }^{1} \mathrm{H}$ NMR (400 MHz, DMSO- $\left.d_{6}\right) \delta$ : $7.62(\mathrm{~s}, 1 \mathrm{H}), 7.37(\mathrm{~s}, 1 \mathrm{H}), 6.88(\mathrm{~s}, 1 \mathrm{H}), 6.52(\mathrm{~s}, 1 \mathrm{H}), 6.13$ $(\mathrm{s}, 1 \mathrm{H}), 5.66(\mathrm{~s}, 1 \mathrm{H}), 4.99(\mathrm{~s}, 1 \mathrm{H}), 4.82 \sim 4.87(\mathrm{~m}, 1 \mathrm{H})$, 4.57 (q, $J=4.0 \mathrm{~Hz}, 1 \mathrm{H}), 2.85$ (d, $J=8.0 \mathrm{~Hz}, 2 \mathrm{H}), 2.06 \sim$ $2.13(\mathrm{~m}, 1 \mathrm{H}), 1.95 \sim 2.00(\mathrm{~m}, 1 \mathrm{H}), 1.21(\mathrm{t}, J=8.0 \mathrm{~Hz}, 3 \mathrm{H})$, $0.83(\mathrm{t}, J=8.0 \mathrm{~Hz}, 3 \mathrm{H}) ;{ }^{13} \mathrm{C}$ NMR $\left(100 \mathrm{MHz}, \mathrm{D}_{2} \mathrm{O}\right) \delta$ : $179.4,164.3,162.0,157.4,144.2,142.1,140.4,128.8$, $128.5,127.2,126.0,105.7,100.7,79.4,55.9,49.3,31.7$, 22.3, 12.4, 7.8; HRMS (ESI) calcd for $\mathrm{C}_{22} \mathrm{H}_{21} \mathrm{~N}_{2} \mathrm{Na}_{2} \mathrm{O}_{6}$ $[\mathrm{M}+\mathrm{H}]^{+}$455.1189, found 455.1190.

参照化合物 SN-38-c 制备方法, 10-HCPT-c 由 10-HCPT 水解制备, 产率 $99 \%$, 为黄色固体. m.p. $>$ $300{ }^{\circ} \mathrm{C}$ (dec.). ${ }^{1} \mathrm{H}$ NMR (400 MHz, DMSO- $\left.d_{6}\right) \delta: 7.72$ (s, $1 \mathrm{H}), 7.58(\mathrm{~d}, J=8.0 \mathrm{~Hz}, 1 \mathrm{H}), 7.37$ (s, 1H), 6.85 (d, $J=8.0$ $\mathrm{Hz}, 1 \mathrm{H}), 6.34$ (s, 1H), 6.09 (s, 1H), 5.59 (s, 1H), 4.95 (s, $2 \mathrm{H}), 4.85$ (q, $J=4.0 \mathrm{~Hz}, 1 \mathrm{H}), 4.58$ (q, $J=4.0 \mathrm{~Hz}, 1 \mathrm{H})$, $2.07 \sim 2.13(\mathrm{~m}, 1 \mathrm{H}), 1.96 \sim 2.01(\mathrm{~m}, 1 \mathrm{H}), 0.83(\mathrm{t}, J=4.0$ $\mathrm{Hz}, 3 \mathrm{H}) ;{ }^{13} \mathrm{C}$ NMR $\left(100 \mathrm{MHz}, \mathrm{D}_{2} \mathrm{O}\right) \delta$ : 179.2, 163.4, $161.7,157.3,145.0,143.1,140.0,130.0,127.5,127.4$, 127.1, 126.1, 109.4, 100.6, 79.5, 55.8, 49.7, 31.6, 7.8; HRMS (ESI) calcd for $\mathrm{C}_{20} \mathrm{H}_{17} \mathrm{~N}_{2} \mathrm{Na}_{2} \mathrm{O}_{6}[\mathrm{M}+\mathrm{H}]{ }^{+}$ 427.0872 , found 427.0877 .

辅助材料(Supporting Information) 化合物附加的吸 收光谱. 这些材料可以免费从本刊网站 (http://siocjournal.cn/)上下载.

\section{References}

[1] (a) Li, C.-J.; Gu, Y.; Han, Y.-X.; Zhou, K. Chin. J. Med. Chem. 2003, 13, 306 (in Chinese). (李春杰, 谷芗, 韩应许, 周凯, 中国药物化学杂志, 2003, 13, 306.)

(b) Schultz, A. G. Chem. Rev. 1973, 73, 385.

(c) Liu, D.; Zhang, L.; Da, F.; Shang, P. J.; Zhang, J.; Yao, L. Prog. Mod. Biomed. 2016, 16, 990 (in Chinese).

(刘丹, 张龙, 达飞, 尚沛津, 张娟, 姚琳, 现代生物医学进展, 2016, 16, 990.)

(d) Chen, L.; Chen, F.-E. Synlett 2017, 28, 1134.

(e) Liu, Y.-Q.; Li, W.-Q.; Morris-Natschke, S. L.; Qian, K.; Yang,
L.; Zhu, G.-X.; Wu, X.-B.; Chen, A.-L.; Zhang, S.-Y.; Nan, X.; Lee, K.-H. Med. Res. Rev. 2015, 35, 753.

(f) Beretta, G. L.; Zuco, V.; De Cesare, M.; Perego, P.; Zaffaroni, N. Curr. Med. Chem. 2012, 19, 3488.

[2] (a) Martino, E.; Della Volpe, S.; Terribile, E.; Benetti, E.; Sakaj, M.; Centamore, A.; Sala, A.; Collina, S. Bioorg. Med. Chem. Lett. 2017, 27, 701

(b) Zunino, F.; Pratesi, G. Exp. Opin. Invest. Drugs 2004, 13, 269.

[3] Pommier, Y. Nat. Rev. Cancer 2006, 6, 789.

[4] (a) Underberg, W. J.; Goossen, R. M.; Smith, B. R.; Beijnen, J. H. J. Pharm. Biomed. Anal. 1990, 8, 681 .

(b) van Warmerdam, L. J.; Verweij, J.; Schellens, J. H.; Rosing, H.; Davies, B. E.; de Boer-Dennert, M.; Maes, R. A.; Beijnen, J. H. Cancer Chemother. Pharmacol. 1995, 35, 237.

(c) Rivory, L. P. Clin. Exp. Pharmacol. Physiol. 1996, 23, 1000. (d) Supko, J. G.; Malspeis, L. J. Liq. Chromatogr. 1991, 14, 1779. (e) Gabr, A.; Kuin, A.; Aalders, M.; El-Gawly, H.; Smets, L. A. Cancer Res. 1997, 57, 4811.

[5] (a) Rowinsky, E. K.; Grochow, L. B.; Hendricks, C. B.; Ettinger, D. S.; Forastiere, A. A.; Hurowitz, L. A.; McGuire, W. P.; Sartorius, S. E.; Lubejko, B. G.; Kaufmann, S. H. J. Clin. Oncol. 1992, 10, 647. (b) Robert, J.; Rivory, L. Drugs Today 1998, 34, 777.

[6] (a) Thomas, C. J.; Rahier, N. J.; Hecht, S. M. Bioorg. Med. Chem. 2004, 12, 1585 .

(b) Oberlies, N. H.; Kroll, D. J. J. Nat. Prod. 2004, 67, 129.

(c) Yuan, Y.; Dong, W.; Gao, X.; Xie, X.; Curran, D. P.; Zhang, Z. Chin. J. Chem. 2018, 36, 1035

(d) Driver, R. W.; Yang, L.-X. Mini-Rev. Med. Chem. 2005, 5, 425.

(e) Curran, D. P.; Josien, H.; Bom, D.; Gabarda, A. E.; Du, W. Ann. N. Y. Acad. Sci. 2000, 922, 112.

[7] (a) Zhang, S. J. Drug Delivery Ther. 2017, 7, 76.

(b) Palakurthi, S. Exp. Opin. Drug Delivery 2015, 12, 1911.

(c) Botella, P.; Rivero-Buceta, E. J. Controlled Release 2017, 247, 28.

(d) Ni, S. J. Drug Delivery Ther. 2017, 7, 73.

(e) Bala, V.; Rao, S.; Boyd, B. J.; Prestidge, C. A. J. Controlled Release 2013, 172, 48 .

(f) Venditto, V. J.; Simanek, E. E. Mol. Pharmaceutics 2010, 7, 307.

[8] (a) Ri, M.; Suzuki, K.; Iida, S.; Hatake, K.; Chou, T.; Taniwaki, M.; Watanabe, N.; Tsukamoto, T. Intern. Med. 2018, 57, 939.

(b) Hamaguchi, T.; Tsuji, A.; Yamaguchi, K.; Takeda, K.; Uetake, H.; Esaki, T.; Amagai, K.; Sakai, D.; Baba, H.; Kimura, M.; Matsumura, Y.; Tsukamoto, T. Cancer Chemother. Pharmacol. 2018, 82, 1021.

[9] Tran, S.; DeGiovanni, P.-J.; Piel, B.; Rai, P. Clin. Trans. Med. 2017 6,44 .

[10] (a) Ko, Y. H.; Kim, E.; Hwang, I.; Kim, K. Chem. Commun. 2007, 1305.

(b) Liu, Y.; Yang, H.; Wang, Z.; Zhang, X. Chem.-Asian J. 2013, 8, 1626.

(c) Tian, J.; Zhang, L.; Wang, H.; Zhang, D.-W.; Li, Z.-T. Supramol. Chem. 2016, 28, 769.

(d) Yang, X.; Liu, F.; Zhao, Z.; Liang, F.; Zhang, H.; Liu, S. Chin. Chem. Lett. 2018, 29, 1560.

(e) Li, T.-T.; Wen, L.-L.; Ji, H.-L.; Liu, F.-Y.; Sun, S.-G. Chin. Chem. Lett. 2017, 28, 463

(f) Bai, D.; Wang, X.; Gao, Z.; Qiu, S.; Tao, Z.; Zhang, J.; Xiao, X Chin. J. Org. Chem. 2017, 37, 2022 (in Chinese)

(白东, 王金鍂, 高中政, 邱胜超，陶朱，张建新，肖昕，有机化学, J. Org. Chem. 2017, 37, 2022.)

(g) Fan, Y.; Lin, F.; Xu, X.-N.; Xu, J.-Q.; Zhao, X. Acta Polym. Sin. 2017, 80 (in Chinese).

(訤显，林沨，徐晓娜，徐甲强，赵新，高分子学报, 2017, 80.)

(h) Yin, H.; Wang, R. Isr. J. Chem. 2018, 58, 188

(i) Yang, B.; Yu, S.-B.; Wang, H.; Zhang, D.-W.; Li, Z.-T. Chem.-Asian J. 2018, 13, 1312.

(j) Zou, H.; Liu, J.; Li, Y.; Li, X.; Wang, X. Small 2018, 14, 1802234 . 
(k) Pazos, E.; Novo, P.; Peinador, C.; Kaifer, A. E.; Garcia, M. D. Angew. Chem., Int. Ed. 2019, 58, 403.

[11] (a) Tian, J.; Wang, H.; Zhang, D.-W.; Liu, Y.; Li, Z.-T. Natl. Sci. Rev. 2017, 4, 426 .

(b) Wang, H.; Zhang, D.-W.; Li, Z.-T. Acta Polym. Sin. 2017, 19 (in Chinese).

(王辉, 张丹维, 黎占亭, 高分子学报, 2017, 19.)

(c) Tian, J.; Chen, L.; Zhang, D.-W.; Liu, Y.; Li, Z.-T. Chem. Commun. 2016, 52, 6351.

(d) Wang, H.; Zhang, D.-W.; Zhao, X.; Li, Z.-T. Acta Chim. Sinica 2015, 73, 471 (in Chinese).

(王辉, 张丹维, 赵新, 黎占亭, 化学学报, 2015, 73, 471.)

(e) Chen, Y.; Huang, F.; Li, Z.-T.; Liu, Y. Sci. China Chem. 2018, $61,979$.

[12] (a) Tian, J.; Zhou, T.-Y.; Zhang, S.-C.; Aloni, S.; Altoe, M. V.; Xie, S.-H.; Wang, H.; Zhang, D.-W.; Zhao, X.; Liu, Y.; Li, Z.-T. Nat. Commun. 2014, 5, 5574

(b) Wu, Y.-P.; Yang, B.; Tian, J.; Yu, S.-B.; Wang, H.; Zhang, D.-W.; Liu, Y.; Li, Z.-T. Chem. Commun. 2017, 53, 13367.

[13] (a) Brunsveld, L.; Folmer, B. J. B.; Meijer, E. W.; Sijbesma, R. P. Chem. Rev. 2001, 101, 4071.

(b) Huang, Z.; Qin, B.; Chen, L.; Xu, J.-F.; Faul, C. F. J.; Zhang, X. Macromol. Rapid Commun. 2017, 38, 1700312.

(c) Xu, J.-F.; Zhang, X. Acta Polym. Sin. 2017, 37 (in Chinese).

(徐江飞, 张希, 高分子学报, 2017, 37.)

(d) Ma, X.; Tian, H. Acta Polym. Sin. 2017, 27 (in Chinese).

(马骧, 田禾, 高分子学报, 2017, 27.)

(e) Yin, Z.-J.; Wu, Z.-Q.; Lin, F.; Qi, Q.-Y.; Xu, X.-N.; Zhao, X. Chin. Chem. Lett. 2017, 28, 1167.

(f) Liu, L. J. Inclusion Phenom. Macrocyclic Chem. 2017, 87, 1.

[14] (a) Tian, J.; Yao, C.; Yang, W.-L.; Zhang, L.; Zhang, D.-W.; Wang,
H.; Zhang, F.; Liu, Y.; Li, Z.-T. Chin. Chem. Lett. 2017, 28, 798.

(b) Yao, C.; Tian, J.; Wang, H.; Zhang, D.-W.; Liu, Y.; Zhang, F.; Li, Z.-T. Chin. Chem. Lett. 2017, 28, 893.

(c) Yang, B.; Zhang, X.-D.; Li, J.; Tian, J.; Wu, Y.-P.; Yu, F.-X.; Wang, R.; Wang, H.; Zhang, D.-W.; Liu, Y.; Zhou, L.; Li, Z.-T. CCS Chem. 2019, accepted.

(d) Zhang, D.-W.; Wang, H.; Li, Z.-T. Chin. Polym. Bull. 2018, 243 (in Chinese).

(张丹维，王辉，黎占亭，高分子通报，2018，243.)

[15] (a) Tian, J.; Xu, Z.-Y.; Zhang, D.-W.; Wang, H.; Xie, S.-H.; Xu, D.-W.; Ren, Y.-H.; Wang, H.; Liu, Y.; Li, Z.-T. Nat. Commun. 2016, 7, 11580 .

(b) Yu, S.-B.; Qi, Q.; Yang, B.; Wang, H.; Zhang, D.-W.; Liu, Y.; Li, Z.-T. Small 2018, 14, 1801037.

[16] (a) Liu, J.; Huang, Y.; Kumar, A. K.; Tan, A.; Jin, S.; Mozhi, A.; Liang, X.-J. Biotechnol. Adv. 2014, 32, 693.

(b) Gillies, E. R.; Goodwin, A. P.; Fréchet, J. M. Bioconjugate Chem. 2004, 15, 1254.

(c) Lu, Y.; Aimetti, A. A.; Langer, R.; Gu, Z. Nat. Rev. Mater. 2016, 2, 16075 .

[17] (a) Zhu, J.; Jin, Z.-M. Pharm. Chem. J. 2017, 51, 491.

(b) Chang, S.; Xie, L.; Ding H.; Nie, Y.; Wu, Y.; He, B.; Gu, Z. Pharm. Nanotechnol. 2013, 1, 115.

(c) Zhang, J. A.; Xuan, T.; Parmar, M.; Ma, L.; Ugwu, S.; Ali, S.; Ahmad, I. Int. J. Pharm. 2004, 270, 93.

(d) Beretta, G. L.; Zunino, F. Biochem. Pharmacol. 2007, 74, 1437.

(e) Oguma, T. J. Chromatogr. B 2001, 764, 49.

(f) Herben, V. M. M.; Ten, B.; Huinink, W. W.; Beijnen, J. H. Clin. Pharmacokinet. 1996, 31, 85.

[18] Yan, M.; Liu, X.-B.; Gao, Z.-Z.; Wu, Y.-P.; Hou, J-L.; Wang, H.; Zhang, D.-W.; Liu, Y.; Li, Z.-T. Org. Chem. Front. 2019, 6, 1698.

(Zhao, X.) 